\title{
Identification and characterization of a novel SCYL3-NTRK1 rearrangement in a colorectal cancer patient
}

\author{
Massimo Milione ${ }^{1}$, Elena Ardini ${ }^{2}$, Jason Christiansen ${ }^{3}$, Emanuele Valtorta ${ }^{4}$, \\ Silvio Veronese ${ }^{4}$, Roberta Bosotti ${ }^{2}$, Alessio Pellegrinelli ${ }^{1}$, Adele Test $^{1}{ }^{1}$, Filippo \\ Pietrantonio ${ }^{5}$, Giovanni Fucà ${ }^{6}, \mathrm{Ge} \mathrm{Wei}^{3}$, Danielle Murphy ${ }^{3}$, Salvatore Siena ${ }^{4,5}$, \\ Antonella Isacchi ${ }^{2}$ and Filippo De Braud ${ }^{5,6}$

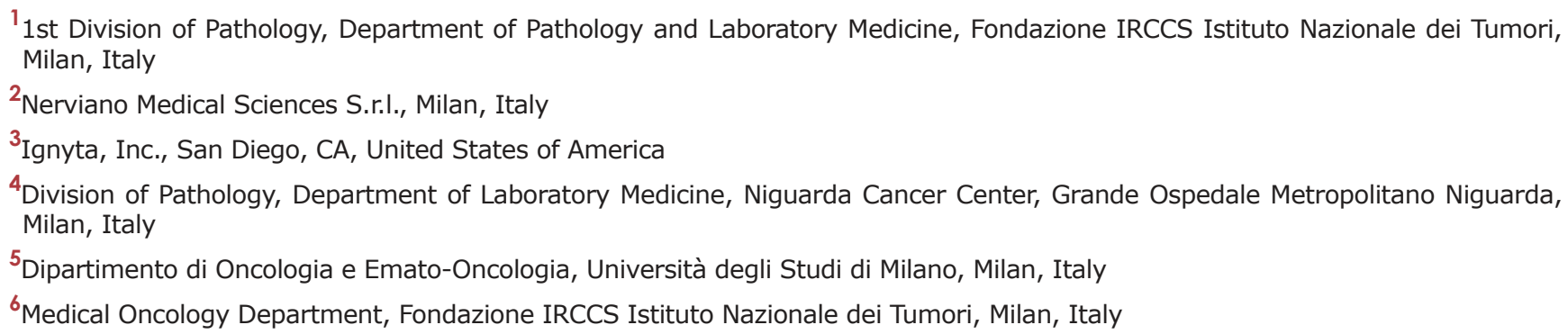

Correspondence to: Massimo Milione, email: Massimo.milione@istitutotumori.mi.it

Keywords: NTRK1, TRKA, entrectinib, CRC, rearrangement

Received: April 12, $2017 \quad$ Accepted: July 12, 2017

Published: July 24, 2017

Copyright: Milione et al. This is an open-access article distributed under the terms of the Creative Commons Attribution License 3.0 (CC BY 3.0 ), which permits unrestricted use, distribution, and reproduction in any medium, provided the original author and source are credited.

\section{ABSTRACT}

In colorectal cancer patients, chromosomal rearrangements involving NTRK1 gene (encoding the TRKA protein) are shown in a small subset of patients and are associated with the constitutive activation of the kinase domain of TRKA. In turn, activated TRKA-fusion proteins are associated with proliferation and survival in colorectal cancer tumors.

Here we report the identification and functional characterization of a new SCYL3NTRK1 fusion gene in a 61-year-old colorectal cancer patient. To our knowledge, this fusion protein has never been previously documented in oncological patients. We show that this novel fusion is oncogenic and sensitive to TRKA inhibitors.

As suggested by other pieces of evidence, entrectinib - an orally available panTRK, ROS1 and ALK inhibitor - may have particular efficacy in patients with NTRK rearrangements. Therefore, screening for rearrangements involving NTRK genes may help identifying a subset of patients able to derive benefit from treatment with entrectinib or other targeted inhibitors.

\section{INTRODUCTION}

Colorectal cancer (CRC) is the third most frequently diagnosed cancer worldwide, with more than one million new cases per year, and represents a major cause of cancerrelated death [1]. Patients with advanced colon cancer are primarily treated with fluoropyrimidine-based systemic chemotherapy with or without irinotecan or oxaliplatin. Despite intense efforts dedicated to the identification of new and more effective therapies, many patients still have a poor treatment outcome. Although targeted agents such as cetuximab, panitumumab, bevacizumab, aflibercept, ramucirumab and regorafenib, introduced into the clinical practice during the last decade, have conferred incremental survival gains $[2,3]$ the identification of novel tumor targets and new targeted therapies is warranted to ensure a better long-term clinical benefit.

We originally reported the identification of a chromosomal rearrangement involving NTRK1 (encoding the TRKA protein) and the TPM3 gene in the KM12 colorectal tumor cell line and showed for the first time that the resulting TPM3-TRKA fusion protein is an oncogenic driver sensitive to TRKA inhibitors. We also showed by screening colorectal tumor samples that this rearrangement is present with approximately $1 \%$ frequency in $\mathrm{CRC}$ and that IHC with anti TRKA antibodies can be successfully 
applied to facilitate the initial screening of rearranged tumors [4]. These findings were further strengthened through genetic screenings performed on large collections of CRC specimens, confirming the presence of NTRK1 rearrangements in $0.5-2 \%$ of CRC patients $[5,6]$. Although many different NTRK1 rearrangements have been already identified in a wide range of solid tumors (see www.ntrkfusions.com), only a limited number of fusion partners has been detected so far in CRC (TPM3-NTRK1, LMNA-NTRK1 and TPR-NTRK1 fusion genes) [5, 7, 8]. All these rearrangements result in the expression of fusion proteins harboring a constitutively activated TRKA kinase domain as a consequence of protein dimerization due to the presence of a coiled-coil domain in the N-terminal sequence of the partner protein. Preclinical data demonstrated that activated TRKA-fusion proteins are associated with/responsible for proliferation and survival in these subsets of CRC tumors $[4,6]$. Importantly, we reported the first evidence of clinical benefit achieved with the TRKA-targeted agent entrectinib in a CRC patient bearing a LMNA-NTRK1 positive tumor, providing clinical validation of activated TRKA as a target in CRC [7].

Based on these promising preclinical data and phase I results, TRKA-selected CRC patients are currently being enrolled in the open-label, multicenter global phase II basket study of entrectinib, an orally available pan-TRK, ROS1 and ALK inhibitor.

The molecular screening, aimed to select TRKApositive patients for the enrollment in the above-mentioned trial, led to the discovery of a CRC patient harboring a novel NTRK1 gene rearrangement consisting of a fusion between the NTRK1 and SCYL3 genes. Here we report the identification and characterization of the resulting SCYL3NTRK1 fusion gene that, to our knowledge, has not been previously reported in cancer patients.

\section{RESULTS}

The patient was a 61-year-old female diagnosed in February 2015 with adenocarcinoma of the right colon, infiltrating the pancreas. The patient progressed early on two standard treatment lines (FOLFOX-panitumumab followed by FOLFIRI-aflibercept). As part of a wide phase I screening program at our institutions (Fondazione IRCCS Istituto Nazionale dei Tumori and Niguarda Cancer Center, Milan, Italy), a deeper molecular characterization of the patient's primary tumor was performed. Results indicated that the tumor was wild type for RAS, BRAF, and EGFR with a high microsatellite instability (MSI-H) profile. At the time of progression to second line therapy (October 2015), the patient underwent endoscopic biopsy of the right-sided tumor mass as part of the pre-screening procedures for the enrollment in the phase I clinical trial ALKA-372-001 (EudraCT Number: 2012.000148-88) [9]. The tumor was tested by immunohistochemistry (IHC) for TRKA, ROS1 and ALK proteins whose expression may indicate the result of a genetic alteration. The IHC analysis revealed strong positivity for TRKA protein with a clear cytoplasmic distribution suggesting a potential aberrancy of NTRK1 gene. The observed immunoreactivity was uniformly characterized by a basic faint cytoplasmic staining associated with a more intense staining organized in irregular or ovoidal clods, preferentially localized around the nuclei (Figure 1A). The size of the clods was highly variable and irregular in shape. Intriguingly, this immunoreactivity pattern appeared very different from the one observed in the patient harboring LMNA-NTRK1 rearrangement, which was a moderate cytoplasmic staining associated with a more intense perinuclear staining as well as from the pattern found in TPM3-NTRK1 bearing KM12 cells, where a diffused cytoplasmic staining could be appreciated (Supplementary Figure 2) [4, 7].

In order to verify if the detected TRKA expression was indeed the result of a genomic rearrangement, fluorescence in situ hybridization (FISH) analysis was performed using a commercial break-apart probe for the NTRK1 gene (Abnova). Results from this analysis showed the presence of break-apart signal, with separate green and orange signals in $90 \%$ of analyzed nuclei, confirming the presence of a NTRK1 rearrangement (Figure 1B). The FISH pattern was similar to the one previously detected in the KM12 cell line.

Since the immunohistochemical staining of our sample was strikingly different from the one previously observed in the KM12 cells, we performed a second FISH analysis with customized break-apart probes (Empire Genomics), initially used for the identification of LMNA-NTRK1 gene fusion in another CRC sample [7]. This further FISH analysis showed that the red centromeric signal from the break-apart probe was absent in $68 \%$ of analyzed nuclei, confirming once again the presence of a genetic alteration involving the NTRK1 gene (Figure 1C). The observed discrepancy between the two hybridization patterns may be due to the specific features of the probes used in the two FISH analyses. In particular the loss of the red-labeled centromeric probe observed in the second analysis only can be explained with the different length of the centromeric probe. Indeed, differences in FISH probe design have previously been shown to complicate the evaluation of the small interstitial deletion that forms the GOPC-ROS1 fusion [10]. Taken together, these observations suggested the presence of a novel NTRK rearrangement and prompted further molecular characterization for the identification of the N-terminal partner gene. An RNA-based anchored multiplex polymerase chain reaction Next Generation Sequencing (NGS) assay, customized for the detection of rearrangements of selected tyrosine kinases and for simultaneous identification of the fusion partner, was applied [11]. This approach allowed the identification of a new NTRK1 rearrangement resulting from an inversion within chromosome 1 fusing exons 1-11 of the SCY1 


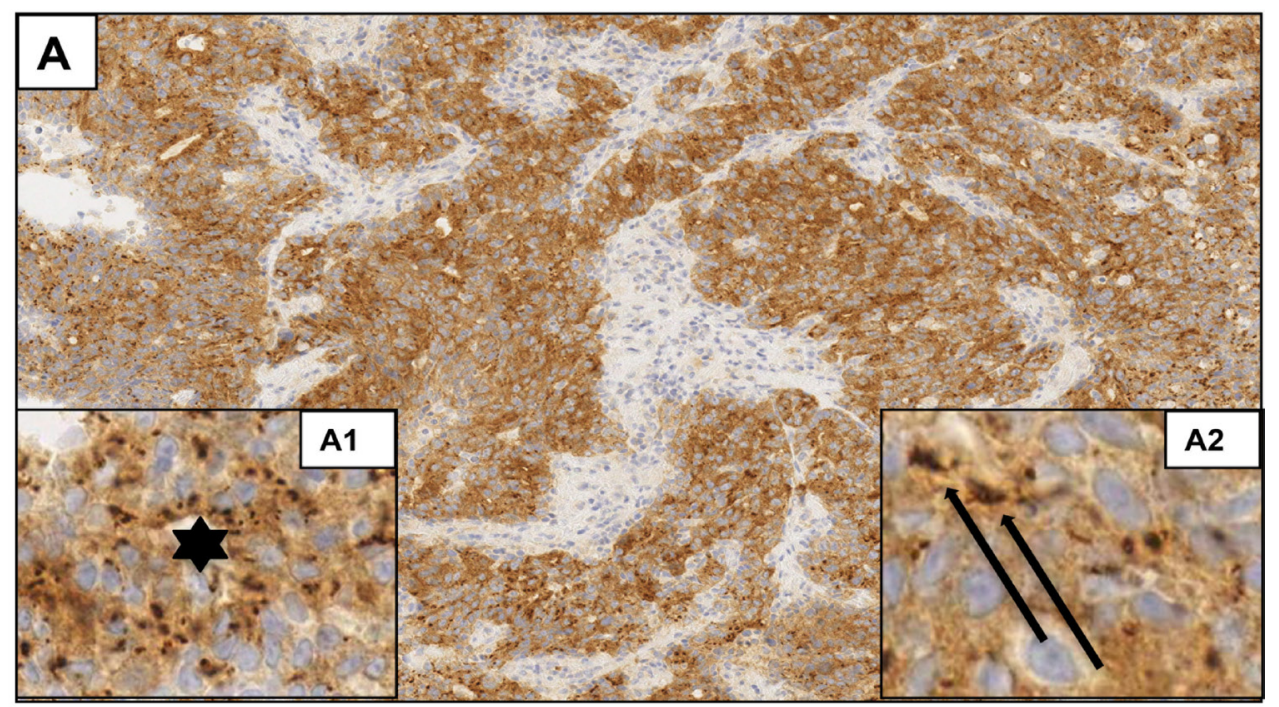

B
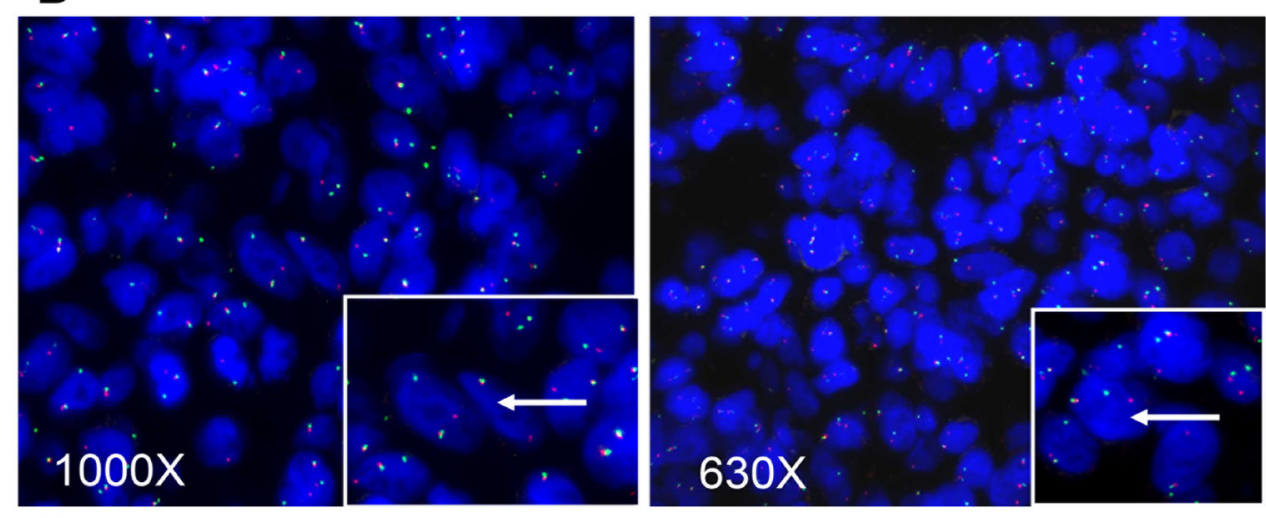

C
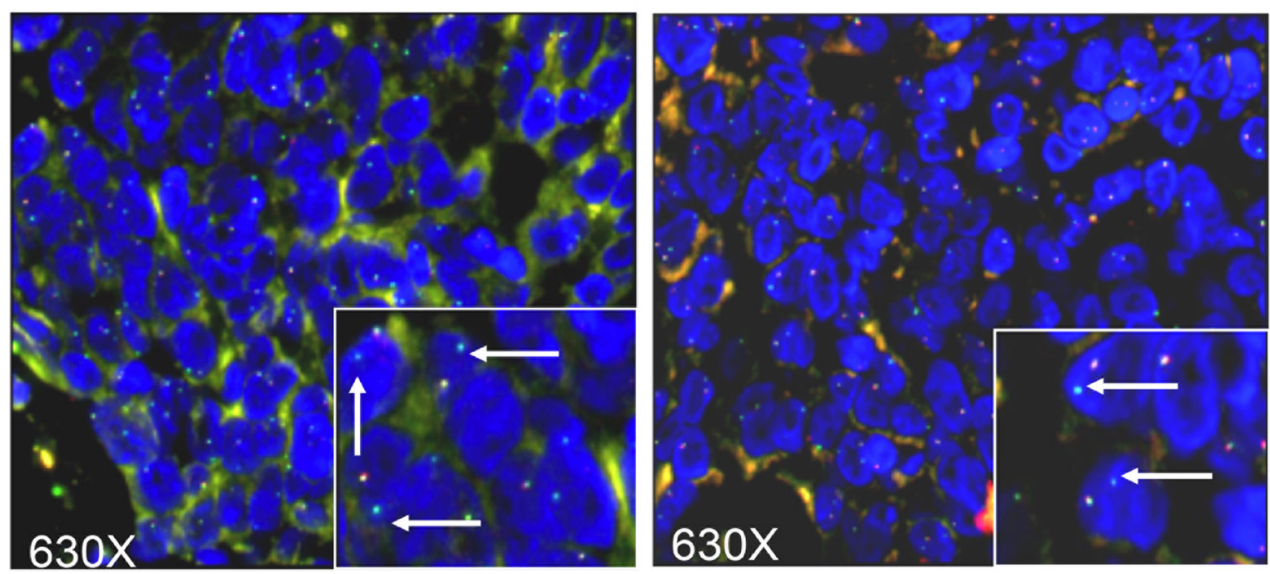

Figure 1: Histologic, immunohistochemical, and fluorescence-in-situ hybridization analyses of the case presented. Immunohistochemical and Fluorescent-In-Situ Hybridization (FISH) images of poorly differentiated CRC. In immunohistochemical assays Panel (A), magnification 100X, whole neoplastic cells are stained by TRKA. The observed staining is characterized by a basic faint uniformly cytoplasmic staining associated to more intense staining organized in irregular round or ovoidal dark bodies (Insert A1, star: magnification 400X) preferentially distributed around nucleus. Dark bodies' size is variable ranging from tiny dot spherules-like to bigger bodies with irregular size and shape. The bigger bodies are fused in coarse ovoid structure surrounding nucleus (Insert A2, arrow; magnification 400X). In panel (B), FISH analysis using the Abnova Break Apart probes showed the presence of one fusion signal along with separate green and orange signals suggesting the presence of a rearrangement of NTRK1 gene. In Panel (C), FISH analysis performed with customized break-apart probes from Empire Genomics showed the green signal only, corresponding to the telomeric part of the break apart probe (white arrows inset), while the red signal corresponding to the centromeric probe (which covers the NTRK1 gene) is missing. Magnification of images: 1000X or 630X as indicated. 
Like Pseudokinase 3 (SCYL3) gene with exons 12-17 of NTRK1 gene (Figure 2). No other NTRK1 fusion transcripts were observed.

As this rearrangement has never been previously described, to formally demonstrate its oncogenic potential, we transfected IL3-dependent $\mathrm{Ba} / \mathrm{F} 3$ cells (a murine pro-B system that does not possess any endogenous human TRKA expression) with SCYL3-NTRK1 cDNA construct (Supplementary Figure 1). As consequence of the expression of the corresponding TRKA-containing fusion protein, $\mathrm{Ba} / \mathrm{F} 3$ cells acquired IL3-independent proliferation capability, demonstrating that SCYL3NTRK1 is indeed an oncogenic driver. These transformed cells were used to test and compare the potency of TRK inhibitors $[6,9]$. Treatment with three different TRK inhibitors strongly affected the proliferation of $\mathrm{Ba} / \mathrm{F} 3-\mathrm{SCYL} 3-N T R K 1$ whereas no growth inhibition was observed in the parental $\mathrm{Ba} / \mathrm{F} 3$ cells or the $\mathrm{Ba} / \mathrm{F} 3$ SCYL3-NTRK1 cells grown in the presence of IL-3 [12]. Entrectinib was found to be the most potent compound inhibiting the proliferation of TRKA-driven cells with an IC50 value of $1.5 \mathrm{nM}$. Other TRK inhibitors LOXO-101 and Crizotinib were also able to decrease the proliferation of $\mathrm{Ba} / \mathrm{F} 3-\mathrm{SCYL} 3-\mathrm{NTRK} 1$ cells with $\mathrm{IC}_{50}$ values of 11.2 $\mathrm{nM}$ and $160 \mathrm{nM}$, respectively (Figure 3A). The mechanism of action of entrectinib was confirmed by flow cytometer analysis of Propidium Iodide (PI) stained cells 18-hour post entrectinib treatment. Consistent with previous publication [13], entrectinib induced cell cycle arrest at G0/G1 (Figure 3C). Furthermore, caspase 3/7 activities were peaked between 24 to 30 hours upon entrectinib treatment, at as low as $3.7 \mathrm{nM}$ (Figure 3D).

Western Blot analysis demonstrated a dose-dependent modulation of TRKA phosphorylation with concomitant inhibition of phosphorylation of the downstream signal transducer PLC $\gamma$ (Figure 3B), consistent with prior studies
[13]. Crizotinib was also able to modulate TRKA signaling, although at significantly higher doses, in agreement with its calculated $\mathrm{IC}_{50}$.

\section{DISCUSSION}

The identification of oncogenic fusions resulting from chromosomal rearrangements in many different tumor types represents an important opportunity for novel therapeutic strategies. This is definitely true for lung adenocarcinoma, as the introduction into the clinical practice of therapeutic approaches based on the specific targeting of activated kinases has dramatically changed the clinical outcome of many patients $[14,15]$. By contrast, in a high un-met medical need such as CRC, the era of targeted therapy coupled with positive predictive biomarkers, is still in its infancy. For many years molecular analyses performed in metastatic CRC patients have been aimed mainly to identify $R A S$ or $B R A F$ mutations that are associated with the lack of response to anti-EGFR treatment $[16,17]$. The identification of positive predictive biomarkers to be exploited for targeted therapeutic approaches in CRC, such as HER2 for dual HER2 blockade, MSI-H for immune checkpoint inhibitors, BRAF for dual/triple inhibition strategies and MGMT methylation for alkylating agents [18-21], represents a paradigm shift for the future treatment landscape [22]. The identification of chromosomal rearrangements involving NTRK1 as a low frequency event in a subset of CRC patients is a relatively recent finding [23]. So far limited efforts have been dedicated to the screening of a high number of cases in order to define the prevalence and the relevance of these findings for CRC patient population. The clinical validation of NTRK1 rearrangements as targets for therapy in CRC will spur the molecular screening of an increasing number of patients, widening

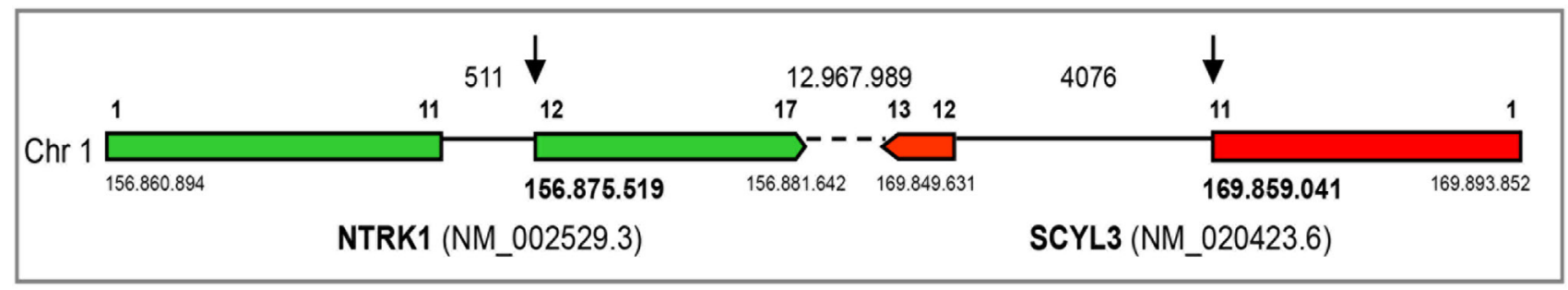

1 1112

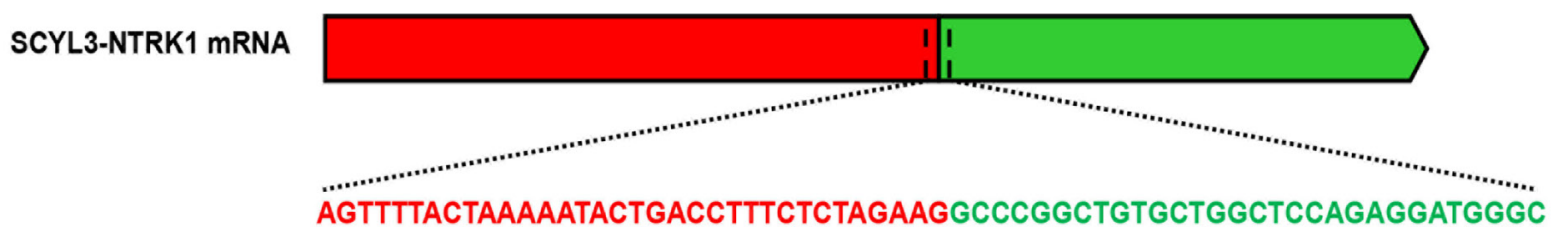

Figure 2: Identification of the SCYL3-NTRK1 gene rearrangement. Schematic representation of the SCYL3-NTRK1 rearrangement. Exons involved in the rearrangement are represented by colored boxes: SCYL3 is reported in green, while NTRK1 is reported in red. The sequence spanning the junction site is shown in detail. 
the opportunity for the application of TRKA-targeted agents into the clinical practice.

In our laboratories, the presence of NTRK1 rearrangements is routinely evaluated by IHC in all CRC tumors in the course screening for patients' enrollment into clinical trials with entrectinib. This screening previously resulted in the identification of two oncogenic fusions, TPM3-NTRK1 and LMNA-NTRK1, that were fully characterized. We also demonstrated that the patients harboring TRK rearrangements derive clinical benefit from the treatment with entrectinib, a specific TRK inhibitor [9]. Here we report the identification of a new chromosomal rearrangement involving NTRK1 that results in the generation of the chimeric SCLY3TRKA fusion protein. Despite the significant number of different fusion partners that has already been identified for NTRK1 in solid tumors, to our knowledge this is the first time that the SCYL3 gene is found to be involved in a rearrangement with NTRK1 or any other kinase gene. It will be interesting to understand if this fusion is specific to CRC or it is also present in other tumor types, such as non small cell lung cancer, papillary thyroid carcinoma and glioblastomas, where multiple NTRK1 rearrangements have already been identified [23-25].

SCYL3, also known as PACE-1, a gene widely expressed in a variety of tissues, encodes for a protein whose function is still poorly understood. The full-length protein is able to bind to ezrin through a C-terminal domain determining its localization at the level of lamellipodia, a specialized cell structure that facilitates cell advancement across a substrate, where it probably plays a role in cell spreading and motility. In addition the N-terminal region contains a myristoylation motif that is responsible for its association with the Golgi apparatus. Intriguingly, SCYL3 protein contains a kinase domain but any attempt to demonstrate its intrinsic kinase activity failed so far, suggesting that it should be probably considered a pseudokinase [26].

As the SCYL3 gene maps to chromosome 1q23.3-4 (1q24.2 on NCBI, OMIM, Gene Cards) we hypothesize
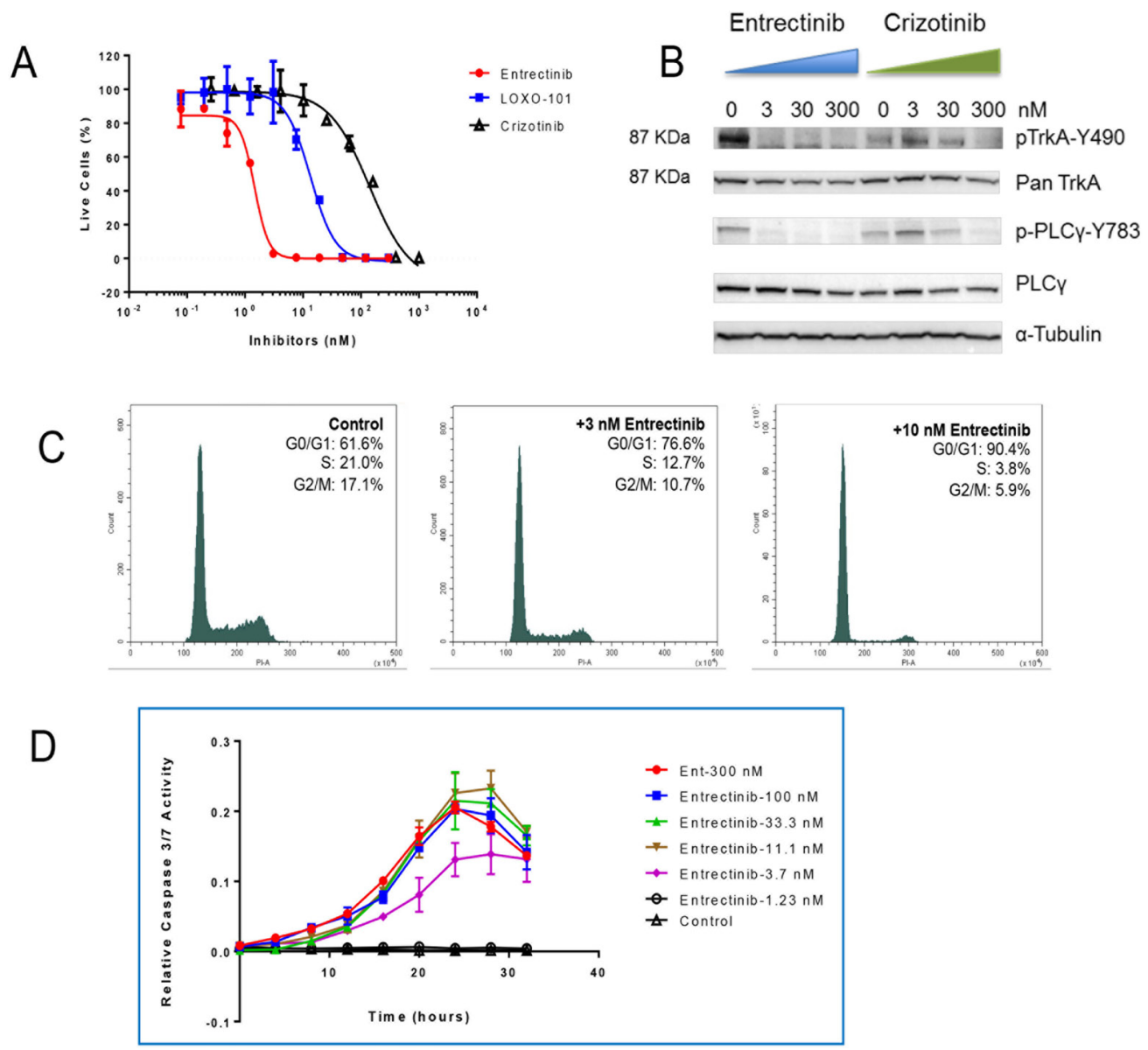

Figure 3: Mechanism of action of entrectinib. Confirmation that the SCYL3-NTRK1 fusion gene can be targeted by TRK inhibitors. (A) Comparison of anti-proliferation activities of 3 tyrosine kinase inhibitors in Ba/F3-SCYL3-NTRK1 cell line. (B) Western blot analysis of the changes in phosphorylation levels of TRKA and its downstream transducer PLC $\gamma 2$ hours post entrectinib and crizotinib treatment in Ba/F3-SCYL3-NTRK1 cells. (C) Cell cycle analysis of Ba/F3-SCYL3-NTRK1 treated with 3 and $10 \mathrm{nM}$ of entrectinib for 18 hours compared to untreated cells. (D) Relative caspase 3/7 activities in Ba/F3-SCYL3-NTRK1 cells treated with increasing concentrations of entrectinib over time. 
that the generation of the fusion gene SCYL3-NTRK1 is a consequence of an intrachromosomal inversion that juxtaposes the first 11 exons of SCYL3 to a portion encompassing exon-12-17 of NTRK1. As a consequence of this fusion event SCYL3 loses the C-terminal sequence responsible for ezrin binding but it maintains its myristoylation motif. The expected localization of the resulting fusion protein SCYL3-TRKA at the level of the Golgi apparatus is compatible with the characteristic punctate intracellular staining pattern observed by immunohistochemical analysis [27]. The in vitro data clearly demonstrate that the SCYL3-NTRK1 fusion gene is oncogenic and that the resulting fusion protein has constitutive kinase activation. Targeted inhibitors, in particular entrectinib, strongly inhibit the SCYL3-TRKA phosphorylation leading to cell growth inhibition and confirming the role of the expressed fusion protein as driver for proliferation. Our data demonstrate that entrectinib represents an important potential opportunity for the treatment of patients whose tumors harbor this new NTRKI rearrangement. Unfortunately, the clinical conditions of this patient rapidly degraded because of tumor progression and it was not possible to treat her with entrectinib.

However, consistent with prior published reports of tumor growth inhibition in NTRK1 rearranged patients treated with entrectinib [7-9], our data showed that this drug has a significant therapeutic potential against newly identified NTRK1 gene rearrangements. Consequently, the screening of $\mathrm{CRC}$ for the detection of gene rearrangements involving the NTRK1, NTRK2 and NTRK3 genes has the ability to identify a limited subset of patients able to derive benefit from treatment with entrectinib or other targeted inhibitors.

\section{MATERIALS AND METHODS}

\section{Immunohistochemistry}

TRKA protein expression was assessed in formalin-fixed paraffin-embedded (FFPE) tumor tissue sections using a rabbit monoclonal antibody (clone EP1058Y; Epitomics, Burlingame, CA) that recognizes the carboxyl-terminus of the protein. Briefly, three micron-thick sections were reacted for $30 \mathrm{~min}$ with the anti-TRKA antibody used at 1:200 working dilution and then incubated with a commercially available detection kit (EnVision ${ }^{\mathrm{TM}}$ FLEX+, Dako, Glostrup, Denmark) following the manufacturer's instructions and previously refined IHC methods. The specificity of all reactions was checked replacing the primary antibody with a non-related mouse immunoglobulin at comparable dilutions or using normal serum alone, Positive and negative controls were used as appropriate KM12 cell line, known to carry a TPM3-NTRK1 gene rearrangement, was used as positive control.

\section{FISH analysis for NTRK1}

In order to identify possible rearrangements involving the NTRK1 gene, dual colour FISH analysis was performed on $3 \mu \mathrm{m}$-thick sections of FFPE tumor tissue using a commercially available, specific break-apart probe kit (Abnova) and following the manufacturer's instructions. In addition customized break-apart probes, (Empire Genomics), previously described, were used [7]. Briefly, NTRK1 FISH analysis was conducted using $10 \mu \mathrm{l}$ mix-probe made up by $1 \mathrm{ul} \mathrm{BAC} \mathrm{(Bacterial}$ Artificial Chromosome) genomic probe RP11-349I17 (1q23.1) (206 kb) labelled in SpectrumOrange (Empire Genomics), 1 ul BAC (Bacterial Artificial Chromosome) genomic probe RP11-1038N13 (1q23.1) (129 kb) labelled in SpectrumGreen (Empire Genomics), 1 ul of sterile water and $7 \mathrm{ul}$ of hybridisation buffer (Empire Genomics). Probes and target DNA of specimen were codenatured in HYBRite System (DakoGlostrup, Denmark) for $5 \mathrm{~min}$ at $75^{\circ} \mathrm{C}$ and then hybridized overnight at $37^{\circ} \mathrm{C}$. Slides were washed with post-hybridisation buffer (DakoGlostrup, Denmark) $\left(73^{\circ} \mathrm{C}\right.$ for $2 \mathrm{~min}$ ) and counterstained with 4,6-diamino-2phenylindole (DAPI II; Vysis, Vysis, Downers Grove, IL. USA). A sample was scored as positive if rearrangements of NTRK1 gene were detected in at least 15 of 100 analysed nuclei. Healthy tissue was used as internal negative control. Fluorescent in situ hybridisation signals were evaluated with a Zeiss Axioscope Imager Z1 (Zeiss,Gottingen, Germany). Images for documentation were captured with CCD camera and processed using the MetaSystems Isis software.

\section{Targeted RNA sequencing}

Gene rearrangements/fusions were analyzed by targeted RNA sequencing, using an anchored multiplex PCR (AMP) method (ArcherDx, Inc.) as previously described [11]. Briefly, a custom panel was generated to focus on determination of gene rearrangements in NTRK1, NTRK2, NTRK3, ROS1, and ALK and RET genes (encoding the TRKA, TRKB, TRKC, ROS1, ALK and RET proteins) along with associated housekeeping genes to assess RNA fragmentation. The AMP method allows for improved sensitivity to novel fusion partners due to an initial adapter ligation step that facilitates priming without de novo knowledge of the gene rearrangement partner and limitations from the small fragments generated in FFPE tissues.

This study was performed according to the clinical standards of the 1975 and 1983 Declaration of Helsinki and was approved by the Ethical Committee of Fondazione IRCCS Istituto Nazionale dei Tumori ( $\mathrm{n}^{\circ}$ INT 48/16). 


\section{Functional studies of SCYL3-NTRK1 fusion gene}

cDNAs (see supplementary data) encoding the SCYL3-NTRK1 fusion protein was inserted into the lentiviral vector $\mathrm{pVL}-\mathrm{EF} 1 \mathrm{a}-\mathrm{MCS}-\mathrm{IRES}-\mathrm{Puro}$ (BioSettia, San Diego, CA) and introduced into the murine, IL-3 dependent, pro-B $\mathrm{Ba} / \mathrm{F} 3$ cells (DSMZ) [12]. $\mathrm{Ba} / \mathrm{F} 3-$ SCYL3-NTRK1 stable cells only proliferate without the need of IL-3. Proliferation assays were conducted with these cells with various concentrations of entrectinib (synthesized by Ignyta), LOXO-101 (synthesized by Ignyta) and crizotinib (purchased from Sellekchem). Plates were incubated at $37^{\circ} \mathrm{C}$ in $5 \% \mathrm{CO}_{2}$ for 72 hours, after which cell viability was assessed by measuring ATP content using Cell Titer-Glo ${ }^{\circledR}$ Luminescent Cell Viability assay (Promega). $\mathrm{IC}_{50} \mathrm{~s}$ were calculated using the 4-parameter variable slope curve fit (GraphPad Prism).

For Western blot analysis, cell lysates were prepared in 1X RIPA buffer (EMDMillipore) with protease and phosphatase inhibitors (EMDMillipore) 2 hours post treatment. The protein lysates were analyzed with SDSPAGE gels (Thermofisher) and transferred to the PVDF membrane using iBlot2 (Thermofisher). The western blot analysis was performed using the primary antibodies (phosphorylated/total TRKA and PLC $\gamma$, and $\alpha$-tubulin) from Cell Signaling Technology and the HRP-labeled secondary antibodies were purchased from LI-COR. The reactive bands were developed by ECL Prime reagent (Amersham) and images were captured by ChemiDoc (Bio-Rad).

To evaluate the effect of entrectinib on the cell cycle, cells were treated with various concentrations of entrectinib for 18 hours and fixed in cold $70 \%$ ethanol, and stained by Propidium Iodide (PI/RNase Staining Buffer (BD Bioscience, cat\#550825)). The cell cycles were analyzed using a CytoFlex flow cytometer (Beckman Coulter).

To confirm the induction of apoptosis by entrectinib, cells were treated with various concentrations of entrectinib and Caspase-3/7 Reagent (IncuCyte, \#4440) was added to all samples. Live images (Phase and green fluorescent channels) were collected at 4-hour intervals over 40 hours. Active Caspase-3/7 activities were normalized to cell numbers.

\section{ACKNOWLEDGMENTS}

Authors would like to acknowledge Patrizia Banfi and Alessio Somaschini for helpful discussion.

\section{CONFLICTS OF INTEREST} to declare

The authors have no potential conflicts of interest

\section{FUNDING}

Authors at Niguarda Cancer Center are supported by AIRC Grant 5x1000 Clinical Molecular Oncology, by Fondazione Oncologia Niguarda Onlus to SS, and by Horizon 2020 MoTriColor Grant.

\section{REFERENCES}

1. International Agency for Research on Cancer. Colon Colorectal Cancer: Estimated Incidence, Mortality and Prevalence Worldwide in 2012 (http://globocan.iarc.fr).

2. Moriarity A, O'Sullivan J, Kennedy J, Mehigan B, McCormick P. Current targeted therapies in the treatment of advanced colorectal cancer: a review. Ther Adv Med Oncol. 2016; 8:276-293.

3. Seeber A, Gastl G. Targeted therapy of colorectal cancer. Oncol Res Treat. 2016; 39:796-802.

4. Ardini E, Bosotti R, Borgia AL, De Ponti C, Somaschini A, Cammarota R, Amboldi N, Raddrizzani L, Milani A, Magnaghi P, Ballinari D, Casero D, Gasparri F, et al. The TPM3-NTRK1 rearrangement is a recurring event in colorectal carcinoma and is associated with tumor sensitivity to TRKA kinase inhibition. Mol Oncol. 2014; 8:1495-1507.

5. Créancier L, Vandenberghe I, Gomes B, Dejean C, Blanchet JC, Meilleroux J, Guimbaud R, Selves J, Kruczynski A. Chromosomal rearrangements involving the NTRK1 gene in colorectal carcinoma. Cancer Lett. 2015; 365:107-111.

6. Park DY, Choi C, Shin E, Lee JH, Kwon CH, Jo HJ, Kim HR, Kim HS, Oh N, Lee JS, Park OK, Park E, Park J, et al. NTRK1 fusions for the therapeutic intervention of Korean patients with colon cancer. Oncotarget. 2016; 7:8399-8412. https://doi.org/10.18632/oncotarget.6724.

7. Sartore-Bianchi A, Ardini E, Bosotti R, Amatu A, Valtorta E, Somaschini A, Raddrizzani L, Palmeri L, Banfi P, Bonazzina E, Misale S, Marrapese G, Leone A, et al. Sensitivity to entrectinib associated with a novel LMNA-NTRK1 gene fusion in metastatic colorectal cancer. J Natl Cancer Inst. 2015; 108.

8. Lee SJ, Li GG, Kim ST, Hong ME, Jang J, Yoon N, Ahn SM, Murphy D, Christiansen J, Wei G, Hornby Z, Lee DW, Park JO, et al. NTRK1 rearrangement in colorectal cancer patients: evidence for actionable target using patientderived tumor cell line. Oncotarget. 2015; 6:39028-39035. https://doi.org/10.18632/oncotarget.5494.

9. Drilon A, Siena S, Ou SI, Patel M, Ahn MJ, Lee J, Bauer TM, Farago AF, Wheler JJ, Liu SV, Doebele R, Giannetta L, Cerea G, et al. Safety and antitumor activity of the multitargeted pan-TRK, ROS1, and ALK inhibitor entrectinib: combined results from two Phase I Trials (ALKA372-001 and STARTRK-1). Cancer Discov. 2017; 7:400-409.

10. Suehara Y, Arcila M, Wang L, Hasanovic A, Ang D, Ito T, Kimura Y, Drilon A, Guha U, Rusch V, Kris MG, Zakowski MF, Rizvi N, et al. Identification of KIF5B-RET and GOPC-ROS1 fusions in lung adenocarcinomas through a comprehensive mRNA-based screen for tyrosine kinase fusions. Clin Cancer Res. 2012; 18:6599-6608.

11. Murphy DA, Ely HA, Shoemaker R, Boomer A, Culver BP, Hoskins I, Haimes JD, Walters RD, Fernandez D, 
Stahl JA, Lee J, Kim KM, Lamoureux J, et al. Detecting gene rearrangements in patient populations through a 2-step diagnostic test comprised of rapid IHC enrichment followed by sensitive next-generation sequencing. Appl Immunohistochem Mol Morphol. 2016.

12. Warmuth $\mathrm{M}, \mathrm{Kim} \mathrm{S}, \mathrm{Gu} X J$, Xia G, Adrián F. Ba/F3 cells and their use in kinase drug discovery. Curr Opin Oncol. 2007; 19:55-60.

13. Ardini E, Menichincheri M, Banfi P, Bosotti R, De Ponti C, Pulci R, Ballinari D, Ciomei M, Texido G, Degrassi A, Avanzi N, Amboldi N, Saccardo MB, et al. Entrectinib, a pan-TRK, ROS1, and ALK inhibitor with activity in multiple molecularly defined cancer indications. Mol Cancer Ther. 2016; 15:628-639.

14. Hirsch FR, Suda K, Wiens J, Bunn PA Jr. New and emerging targeted treatments in advanced non-small-cell lung cancer. Lancet. 2016; 388:1012-1024.

15. Katayama R, Lovly CM, Shaw AT. Therapeutic targeting of anaplastic lymphoma kinase in lung cancer: a paradigm for precision cancer medicine. Clin Cancer Res. 2015; 21:2227-2235.

16. Pietrantonio F, Cremolini C, Petrelli F, Di Bartolomeo M, Loupakis F, Maggi C, Antoniotti C, de Braud F, Falcone A, Iacovelli R. First-line anti-EGFR monoclonal antibodies in panRAS wild-type metastatic colorectal cancer: A systematic review and meta-analysis. Crit Rev Oncol Hematol. 2015; 96:156-166.

17. Pietrantonio F, Petrelli F, Coinu A, Di Bartolomeo M, Borgonovo K, Maggi C, Cabiddu M, Iacovelli R, Bossi I, Lonati V, Ghilardi M, de Braud F, Barni S. Predictive role of BRAF mutations in patients with advanced colorectal cancer receiving cetuximab and panitumumab: a metaanalysis. Eur J Cancer. 2015; 51:587-594.

18. Sartore-Bianchi A, Trusolino L, Martino C, Bencardino K, Lonardi S, Bergamo F, Zagonel V, Leone F, Depetris I, Martinelli E, Troiani T, Ciardiello F, Racca P, et al. Dual-targeted therapy with trastuzumab and lapatinib in treatment-refractory, KRAS codon 12/13 wild-type, HER2positive metastatic colorectal cancer (HERACLES): a proof-of-concept, multicentre, open-label, phase 2 trial. Lancet Oncol. 2016; 17:738-746.
19. Le DT, Uram JN, Wang H, Bartlett BR, Kemberling H, Eyring AD, Skora AD, Luber BS, Azad NS, Laheru D, Biedrzycki B, Donehower RC, Zaheer A, et al. PD-1 blockade in tumors with mismatch-repair deficiency. N Engl J Med. 2015; 372:2509-2520.

20. Pietrantonio F, Oddo D, Gloghini A, Valtorta E, Berenato R, Barault L, Caporale M, Busico A, Morano F, Gualeni AV, Alessi A, Siravegna G, Perrone F, et al. MET-driven resistance to dual EGFR and BRAF blockade may be overcome by switching from EGFR to MET inhibition in BRAF-mutated colorectal cancer. Cancer Discov. 2016; 6:963-971.

21. Pietrantonio F, Perrone F, de Braud F, Castano A, Maggi C, Bossi I, Gevorgyan A, Biondani P, Pacifici M, Busico A, Gariboldi M, Festinese F, Tamborini E, et al. Activity of temozolomide in patients with advanced chemorefractory colorectal cancer and MGMT promoter methylation. Ann Oncol. 2014; 25:404-408.

22. Cremolini C, Pietrantonio F. How the lab is changing our view of colorectal cancer. Tumori. 2016; 102:541-547.

23. Amatu A, Sartore-Bianchi A, Siena S. NTRK gene fusions as novel targets of cancer therapy across multiple types. ESMO Open. 2016; 1:e000023.

24. Vaishnavi A, Capelletti M, Le AT, Kako S, Butaney M, Ercan D, Mahale S, Davies KD, Aisner DL, Pilling AB, Berge EM, Kim J, Sasaki H, et al. Oncogenic and drugsensitive NTRK1 rearrangements in lung cancer. Nat Med. 2013; 19:1469-1472.

25. Greco A, Miranda C, Pierotti MA. Rearrangements of NTRK1 gene in papillary thyroid carcinoma. Mol Cell Endocrinol. 2010; 321:44-49.

26. Kim J, Lee Y, Cho HJ, Lee YE, An J, Cho GH, Ko YH, Joo KM, Nam DH. NTRK1 fusion in glioblastoma multiforme. PLoS One. 2014; 9:e91940.

27. Sullivan A, Uff CR, Isacke CM, Thorne RF. PACE-1, a novel protein that interacts with the C-terminal domain of ezrin. Exp Cell Res. 2003; 284:224-238. 